\title{
The Neuroinvasive Potential of SARS-CoV-2 on the Central Nervous System (lesions in the brain)
}

\author{
Samir Djemli*, Abdelkrim Tahraoui, Mabrouk Boussena, Faiza Rouag, Ines Refes, Samia Messikh, Ibtissem \\ Chouba, Mohamed Kouadria and Rouya Memouni
}

Applied Neuroendocrinology Laboratory, Department of Biology, Faculty of Sciences, Badji Mokhtar Annaba University Algeria.

${ }^{*}$ Corresponding authors

Samir Djemli, Applied Neuroendocrinology Laboratory, Department of Biology, Faculty of Sciences, Badji Mokhtar Annaba University Algeria

Submitted: 21 March2021;Accepted: 30 March 2021;Published: 05 Apr 2021

Citation: Samir Djemli*, Abdelkrim Tahraoui, Mabrouk Boussena, Faiza Rouag, Ines Refes, et al. (2021) The Neuroinvasive Potential of SARS-CoV-2 on the Central Nervous System (lesions in the brain). Medical \& Clinical Research 6(4): 518-519.

\section{Editorial}

The coronavirus that causes Covid-19 could infect the central nervous system like other coronaviruses. Is this related to the cases of anosmia reported by doctors among infected people?

The more than 340,000 people suffering from Covid-19 complain of a triad of symptoms typical of infection: fever, cough, and difficulty breathing [1]. But some also complain of rarer ailments like headaches, nausea and vomiting. These symptoms are usually associated with a neurological condition. Are they witnessing the invasion by the coronavirus responsible for Covid-19 of the central nervous system?

\section{Coronavirus Neurotropism}

Although coronaviruses are essentially respiratory viruses, several studies show their ability to also infect the central nervous system and cause neurological disorders [2]. The neurotropism of coronaviruses is known, in particular for the beta-coronavirus family to which SARS-CoV, MERS-CoV and SARS-CoV-2 belong, which is currently spreading. Neurons in the central nervous system are often the cell targeted by the virus that causes their degeneration. HEV $67 \mathrm{~N}$, a porcine coronavirus, is the first to be identified in the brains of pigs. There is $91 \%$ homology between this strain and a human beta-coronavirus, $\mathrm{HCoV}-\mathrm{OC} 43$, responsible for the common cold [8-10]. The path taken by coronaviruses is not precisely known, but it seems that viruses use synaptic pathways to pass from the cardiorespiratory center to the spinal cord. The mechanoreceptors and chemoreceptors of peripheral nerve endings that are in the lower respiratory tract are said to be the gateway through which the virus enters the central nervous system [3]. The neurological symptoms of Covid-19 only affect a minority of people: $8 \%$ suffer from headaches and 1\% from nausea and vomiting. In contrast, a study of 240 patients with Covid-19 describes neurological manifestations such as loss of consciousness and acute cerebrovascular disorders in $88 \%$ of the severe cases studied [4].

\section{An Explanation for The Anosmia?}

In recent days, doctors have reported that a minority of patients complain of anosmia. It is a loss of smell often associated with a loss of taste (ageusia). Anosmia can be caused by damage to the olfactory nerve, the first cranial nerve that connects the nasal fossa with the olfactory bulb located at the base of the brain. When the attack is neurological, anosmia can be constant [5]. In the case of Covid-19, is anosmia the witness of the invasion of the virus of the central nervous system?

Not necessarily. Anosmia is a very common symptom in respiratory diseases like rhinitis or the common cold. In this case, it is not the olfactory nerve that is involved but a lack of permeability of the nasal cavities, the nose in short, which prevents odors from reaching the olfactory receptors. The anosmia is then generally transient [6]. The virus has not yet been observed in the brainstem of patients, as was the case in tests carried out in mice with MERS-CoV and SARS-CoV. The neurotropism of SARS-CoV-2, responsible for Covid-19, is for the moment only a guess and scientific data is lacking to attest it with certainty. But being aware of this possibility could have an impact on the care and treatment, which are always symptomatic of the disease [7]. Covid-19 can have effects on the brain: stroke, confusion, loss of spatio-temporal landmarks, memory impairment, loss of taste and smell ... Until now, these effects seemed temporary. But Australian scientists now fear that they will cause a series of neurological pathologies in the longer term [8]. Studies are increasing to better understand how SARSCoV-2 impacts the brains of patients. Covid-19 is responsible for specific symptoms - breathing difficulties, fever, dry cough. But neurological signs have also been observed, such as: headaches, delusions or confusions, cognitive dysfunction, fatigue and of course, loss of smell (anosmia) and taste. The disease could even cause strokes [9].

\section{Can The Coronavirus Really Reach the Brain?}

It is not impossible. During the SARS epidemic (also caused by a coronavirus, in 2002), scientists were able to demonstrate 
that particles of the virus could migrate to the brain. In the case of Middle East respiratory syndrome (another member of the coronavirus family, responsible for an epidemic in 2012-2017), the virus was even detected in mice at the neurological level ... without being present in lungs!

How to Explain This Neurological Damage (Alzheimer disease)? The hypothesis currently used by researchers is that of the cytokine storm [10]. In short: faced with the Sars-Cov-2 infection, the body would trigger a disproportionate defense reaction: too extreme, it could lead to "overheating" of the brain ... and specific neurological symptoms $[3,4]$. Sometimes "the virus attacks the brain, although it has not been demonstrated today", sometimes there is irritation of the brain "by" crossing the virus of the bone barrier between nasal cavity and brain "and finally it can there may be "changes in cerebrovascular circulation" [11]. Beyond the coronavirus, mental confusion, disorientation, abnormal agitation, vertigo that does not "pass", convulsions or even nerve pain (tingling, electric shocks ...) can betray the presence of meningitis or even a stroke $[6,7]$.

This fear is based on the memory of a previous epidemic, that of the Spanish flu in 1918/1919. As a result, many cases of lethargic encephalitis appeared. And this viral infection had left as a sequel, in a very large percentage of affected patients, serious disorders, very similar to those of Parkinson's disease (post-encephalitic Parkinsonism).8Since the Spanish flu epidemic, no virus has appeared that attacks the brain in this way. Moreover, most Parkinson's patients suffer from a degenerative disease, the origin of which is not a virus but a process of degeneration [6].

What is the relationship between Parkinson's and Covid-19? Parkinson's disease consists of damage and progressive disappearance of neurons that release dopamine. Secondary (or atypical) Parkinsonism is due to a blocking mechanism or a disturbance in the action of dopamine in the basal ganglia. When the body defends itself against SARS-CoV-2, it causes inflammation in the brain. This could, in the long term, be responsible for this blockade of dopamine, or more generally for neurodegenerative diseases, as was the case in 1920. A first case of this type could have been detected in Israel. But for now, nothing has been formally confirmed.11Finally, the researchers give the potential long-term neurological consequences of COVID-19. If they have no proof, however, they ask that urgent measures be taken to have more precise diagnostic tools allowing the early identification of neurodegeneration.

\section{References}

1. Burks JS, Devald BL, Jankovsky LD, Gerdes JC (1980) Two coronaviruses isolated from central nervous system tissue of two multiple sclerosis patients. Science 209: 933-934.

2. Abbott NJ (2002) Astrocyte-endothelial interactions and blood-brain barrier permeability. Journal of Anatomy 200: 629- 638 .

3. Agosti E, Giorgianni AD, Amore F, Vinacci G, Balbi S, et.al (2020) Is Guillain-Barrè syndrome triggered by SARS-CoV-2 ? Case report and literature review. Neurological Sciences 42: 607-612.

4. Agrawal AS, Garron T, Tao X, Peng BH, Wakamiya M, et al. (2015) Generation of a transgenic mouse model of middle east respiratory syndrome coronavirus infection and disease. Journal of Virology 89: 3659-3670.

5. Altin F, Cingi C, Uzun T, Bal C (2020) Olfactory and gustatory abnormalities in Covid-19 cases. European Archives of OtoRhino-Laryngology 277: 2775-2781.

6. Asadi-Pooya AA, Simani L (2020) Central nervous system manifestations of Covid-19 A systematic review. Journal of the Neurological Sciences 413: 116832.

7. Baig AM, Khaleeq A, Ali U, Syeda H (2020) Evidence of the Covid-19 virus targeting the CNS Tissue distribution hostvirus interaction and proposed neurotropic mechanisms. ACS Chemical Neuroscience 11: 995-998.

8. Bongetta D, Calloni T, Colombo EV, Versace A, Assietti R (2020) Do matrix metalloproteases mediate the sars-cov-2related damage to the central nervous system ? j Immunity 88: 35 .

9. Brann DH, Tsukahara T, Weinreb C, Lipovsek M, Vanden Berge, et al. (2020) Non-neuronal expression of SARS-CoV-2 entry genes in the olfactory system suggests mechanisms underlying COVID-19-associated anosmia. Science Advances 6: 5801 .

10. Bullen CK, Hogberg HT, Bahadirli-Talbott A, Bishai WR, Hartung T, et al. (2020) Infectability of human brainsphere neurons suggests neurotropism of SARS-CoV-2 ALTEX 37: 665-671.

11. Chen G, Wu DI, Guo W, Cao Y, Huang DA, et al. (2020) Clinical and immunological features of severe and moderate coronavirus disease 2019. Journal of Clinical Investigation 130: $2620-2629$.
Copyright: (C2021 Samir Djemli, et al. This is an open-access article distributed under the terms of the Creative Commons Attribution License, which permits unrestricted use, distribution, and reproduction in any medium, provided the original author and source are credited. 